\title{
Is the Association Between Smoking and Depression Mediated by Inflammation? A Mendelian Randomization Study.
}

\author{
Galan, D. ${ }^{1}$, Perry, B.I. ${ }^{2,3}$, Warrier, V. ${ }^{2,3}$, Easton, D. ${ }^{1}$, Khandaker, G.M. ${ }^{2,3,4,5,6}$, Murray, G.K. ${ }^{2,3,7}$ \\ ${ }^{1}$ Department of Public Health and Primary Care, University of Cambridge, Cambridge, UK \\ ${ }^{2}$ Department of Psychiatry, University of Cambridge, Cambridge, UK \\ ${ }^{3}$ Cambridgeshire and Peterborough NHS Foundation Trust, Cambridge, UK \\ ${ }^{4} \mathrm{MRC}$ Integrative Epidemiology Unit, Population Health Sciences, Bristol Medical School, \\ University of Bristol, Bristol, UK \\ ${ }^{5}$ Centre for Academic Mental Health, Population Health Sciences, Bristol Medical School, \\ University of Bristol, Bristol, UK \\ ${ }^{6}$ Avon and Wiltshire Mental Health Partnership NHS Trust, Bristol, UK \\ ${ }^{7}$ Program in Complex Trait Genomics, Institute of Molecular Bioscience, University of \\ Queensland, Brisbane, Australia
}


medRxiv preprint doi: https://doi.org/10.1101/2021.02.04.21251136; this version posted February 8, 2021. The copyright holder for this preprint (which was not certified by peer review) is the author/funder, who has granted medRxiv a license to display the preprint in perpetuity.

It is made available under a CC-BY 4.0 International license .

\begin{abstract}
Smoking, inflammation and depression commonly co-occur and may be mechanistically linked. However, key questions remain around the direction of association and the influence of residual confounding. We aimed to characterize the association between lifetime smoking and depression, as well as to assess the role that genetically-predicted C-reactive protein (CRP) level, an archetypal inflammatory marker, as a potential mediator for this association. We performed inverse variance weighted Mendelian randomization (MR) analyses using recently published summary-level GWAS data for lifetime smoking index, CRP levels, and depression. A subset of inflammatory-related genetic variants from the lifetime smoking GWAS were also used to assess the potential inflammatory causal pathways between smoking and depression. The analysis indicated significant reciprocal relationships between lifetime smoking and both depression $\left(\mathrm{OR}_{\text {Smk-Dep }}=2.01,95 \%\right.$ CI 1.71- 2.37, $\mathrm{p}<0.001 ; \mathrm{O}_{\text {Dep-Smk }}=1.09,95 \%$ CI 1.06- 1.13, $\mathrm{p}<$ $0.001)$ and CRP levels $\left(\mathrm{OR}_{\text {Smk-CRP }}=1.40,95 \%\right.$ CI 1.21-1.55, $\mathrm{p}<0.001 ; \mathrm{OR}_{\mathrm{CRP}-\mathrm{Smk}}=1.03,95 \%$ CI 1.02- 1.05, $\mathrm{p}<0.001)$. These significant and positive associations were also supported by the majority of the robust MR methods performed. The reciprocal relationships between CRP levels (using $>500$ genetic instruments for CRP) and depression were not significant (OR $\mathrm{ORP}_{\text {Cep }}=1.01$,

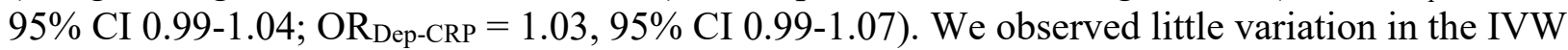
estimates between smoking and depression when we limited the genetic variants assessed to those related to inflammation or when we adjusted the analysis by CRP-levels in multivariable analysis. Our study supports potential causal associations between lifetime smoking and depression, as well as between lifetime smoking and CRP levels, but not between CRP and depression. No evidence was found that CRP mediates the relationship between smoking and depression.
\end{abstract}




\section{Introduction:}

Increasing evidence indicates a role for inflammation in the pathogenesis of mental health disorders, particularly depression ${ }^{1,2}$. Even though, historically, the central nervous system (CNS) has been considered an immuno-privileged region in the human body, research has shown that microglia in the CNS produce inflammatory cytokines and inflammatory processes outside of the CNS can result inflammatory responses within the $\mathrm{CNS}^{3-8}$. Furthermore, diseases associated with inflammation, such as rheumatoid arthritis, diabetes mellitus, coronary heart disease, stroke have been associated with depression ${ }^{9,10}$.

Modifiable exposures, such as smoking behavior, have been associated with both depression and inflammation. A 2014 study in the US estimated that the smoking prevalence among participants $16 \%$ and $40 \%$ in patients without a psychiatric and those who had a diagnosis of MDD during the previous year, respectively ${ }^{11}$. Furthermore, observational studies seem to support a systemic elevation of serum inflammatory markers in smokers ${ }^{12}$, including significant increases in Creactive protein (CRP) levels ${ }^{13-15}$. However, the evidence regarding direction of association and causality is unclear due to the biases inherent to observational studies ${ }^{16,17}$.

Mendelian randomization (MR) is an epidemiological approach that uses genetic variants as instruments to untangle the problems of reverse causation (genetic variants are fixed at conception; hence, genetically predicted levels of risk factors must precede any event) and unmeasured confounding (genetic variants show considerably less conventional confounding than phenotypic variables) ${ }^{18}$. If genetically-predicted values of a risk factor are associated with a specific disease outcome, then it is likely that the association between the risk factor and outcome has a causal basis ${ }^{19-22}$. In this manner, MR studies act like natural randomized control trials and overcome some of the biases of observational studies ${ }^{23}$.

Previous studies have shown through MR analyses that smoking and depression may have a bidirectional causal relationship ${ }^{24}$, although the mechanism through which smoking causes depression is not known. MR studies using CRP as a proxy for systemic inflammation have shown conflicting evidence regarding the association between higher CRP levels and risk for depression ${ }^{20,25,26}$. Recently, statistically better powered genome wide association studies (GWAS) for depression ${ }^{27}$ and for $\mathrm{CRP}^{28}$ have become available, and provide an opportunity to use improved genetic instruments, which explain a larger proportion of the variable's variance, for causal inference to resolve prior ambiguities.

In this study, first to examine potential causality and direction of association, we conducted univariable and bidirectional MR analysis testing associations of genetically-predicted smoking behavior with CRP levels and risk of depression, and vice versa. Second, to examine for a potential mediating role of inflammation between smoking behavior and risk of depression, we conducted univariable MR analyses limiting the smoking exposure genetic variants to those which have been previously associated with inflammatory traits. Finally, we conducted a multivariable Mendelian randomization (MVMR) analysis to examine the associations between 
medRxiv preprint doi: https://doi.org/10.1101/2021.02.04.21251136; this version posted February 8, 2021. The copyright holder for this preprint (which was not certified by peer review) is the author/funder, who has granted medRxiv a license to display the preprint in perpetuity.

It is made available under a CC-BY 4.0 International license.

genetically-predicted smoking behavior and risk of depression after adjusting for geneticallypredicted CRP levels using CRP levels to adjust the effect of the smoking genetic variants on depression. We used the latest GWAS data to develop statistically better powered genetic instruments compared to previous studies and used this to investigate if inflammation mediates the potential causal effect of smoking on depression. 


\section{Methods:}

\section{Data Sources:}

This study used publicly available summary level data obtained from previously published genome-wide association studies (GWAS) for all analyses. The source and description of the GWAS summary statistics used in this report for lifetime smoking, depression, and C-reactive protein (CRP) are presented in Table 1.

Table 1. Characteristics of the GWAS from which the summary statistics were obtained

\begin{tabular}{|l|l|l|l|l|l|l|}
\hline Variable & $\begin{array}{l}\text { GWAS } \\
\text { Source }\end{array}$ & $\begin{array}{l}\text { Population } \\
\text { Used }\end{array}$ & $\begin{array}{l}\text { Total } \\
\text { Participants }\end{array}$ & Cases & Controls & $\begin{array}{l}\text { SNPs } \\
\text { Covered }\end{array}$ \\
\hline $\begin{array}{l}\text { Lifetime } \\
\text { Smoking }\end{array}$ & Wootton et & $\begin{array}{l}\text { UK } \\
\mathrm{al}^{24}\end{array}$ & 462,690 & 249,318 & 213,372 & $7,683,352$ \\
\hline Biobank & $\begin{array}{l}\text { UK } \\
\text { Biobank \& } \\
33 \text { cohorts }\end{array}$ & PGC & 500,199 & 170,756 & 329,443 & $8,483,301$ \\
\hline $\begin{array}{l}\text { C-Reactive } \\
\text { Protein } \\
\text { Levels }\end{array}$ & Han et al ${ }^{28}$ & $\begin{array}{l}\text { UK } \\
\text { Biobank }\end{array}$ & 418,642 & NA & NA & $8,927,092$ \\
\hline
\end{tabular}

\section{Lifetime Smoking Index:}

Lifetime smoking index was selected as the variable to represent the smoking exposure for all analyses. Wootton et al. (2019) generated this lifetime smoking index, encompassing information regarding smoking heaviness, duration, and smoking initiation and cessation. One standard deviation increase in lifetime smoking score is equivalent to an individual smoking 20 cigarettes a day for 15 years and stopping 17 years ago or an individual smoking 60 cigarettes a day for 13 years and stopping 22 years ago $^{24}$.

\section{Depression:}

Depression summary statistics were obtained from the Psychiatric Genetic Consortium (PGC). These data combined the meta-analysis efforts of two previously published articles ${ }^{27,29}$. The resulting summary statistics include a broad definition of depression, as they encompass the diagnostic criteria used as case definitions in the different cohorts included (Table 2).

Table 2. Depression case definitions used in the different cohorts combined for the depression GWAS summary statistics from the PGC ${ }^{27,29}$.

\begin{tabular}{|l|l|l|}
\hline Study & Cohort & Depression Definition \\
\hline $\begin{array}{l}\text { Howard et al. } \\
(2019)\end{array}$ & UK Biobank & $\begin{array}{l}\text { (1) Self-reported past help- } \\
\text { seeking for problems with } \\
\text { "nerves, anxiety, tension or } \\
\text { depression", (2) self-reported } \\
\text { depressive symptoms with }\end{array}$ \\
\hline
\end{tabular}




\begin{tabular}{|l|l|l|}
\hline & & $\begin{array}{l}\text { associated impairment, and } \\
\text { (3) MDD identified from } \\
\text { ICD-9 or ICD-10-coded } \\
\text { hospital admission records. }\end{array}$ \\
\hline Wray et al. (2018) & PGC29* & $\begin{array}{l}\text { Structured diagnostic } \\
\text { interviews. }\end{array}$ \\
\hline & deCODE & Electronic medical records. \\
\hline & GenScotland & $\begin{array}{l}\text { Structured diagnostic } \\
\text { interviews. }\end{array}$ \\
\hline & GERA & $\begin{array}{l}\text { Electronic medical records. } \\
\text { Electronic medical records. }\end{array}$ \\
\hline & iPSYCH & $\begin{array}{l}\text { (1) Self-reported MDD } \\
\text { symptoms, (2) self-reported } \\
\text { MDD treatment, or (3) } \\
\text { electronic medical records. }\end{array}$ \\
& UK Biobank ${ }^{+}$ & $\begin{array}{l}\text { Self-reported diagnosis or } \\
\text { treatment for clinical } \\
\text { depression by a medical } \\
\text { professional }\end{array}$ \\
\hline & 23andMe & \\
\hline & &
\end{tabular}

${ }^{*} P G C 29$ includes 29 different cohorts ${ }^{30}$.

${ }^{+}$UK Biobank samples were excluded to prevent overall with Howard et al. (2019).

\& 23andMe samples were excluded from the publicly available results.

\section{C-reactive Protein:}

C-reactive protein (CRP) was selected as the proxy for systemic inflammation ${ }^{6,14,15,31,32}$. Han et al. (2019) generated the summary statistics used for this study from 418,642 individuals of British ancestry in the UK biobank for whom CRP levels were available and whose levels were lower than $10 \mathrm{mg} / \mathrm{L}^{28}$.

\section{Selection of Genetic Instrumental Variable:}

In Mendelian randomization, for a genetic variant to be a valid IV, it must meet three assumptions: (i) the variant is associated with the exposure, (ii) the variant is not associated with any confounder of the exposure-outcome association,(iii) the variant does not affect the outcome, except possibly via its association with the exposure ${ }^{33}$. The process for IV selection from the GWAS summary statistics for each of the variables studied was performed following a primarily statistical approach ${ }^{34}$. However, in some cases, the IV selection was further refined to include biological factors, as described below.

\section{Statistical selection:}

SNPs were considered significantly associated to the GWAS variable of interest if the GWAS pvalue reported on the summary statistics was smaller than $5 \times 10^{-8}{ }^{33}$. Using multiple correlated variants representing the same effect would decrease the efficiency of the analyses and increase the risk of weak instrument bias in the estimates obtained without increasing the power of the study ${ }^{35,36}$. Consequently, absence of LD and independence of the final IVs selected was 
medRxiv preprint doi: https://doi.org/10.1101/2021.02.04.21251136; this version posted February 8, 2021. The copyright holder for this preprint (which was not certified by peer review) is the author/funder, who has granted medRxiv a license to display the preprint in perpetuity.

It is made available under a CC-BY 4.0 International license .

ascertained using the $l d$ clump() function from the ieugwasr $\mathrm{R}$ package (clumping window $\mathrm{kb}=$ $\left.10000, r^{2}=0.001, \mathrm{p}=0.99\right)^{37}$.

If an IV selected did not have a match in the outcome GWAS statistics, a proxy IV (in linkage disequilibrium with the original IV; $r^{2}>0.8$ ), was used instead. Proxy IVs were obtained using the LDlink R package ${ }^{38}$.

\section{Biological selection:}

Smoking inflammatory SNPS:

Out of the lifetime smoking IVs determined significant and in linkage disequilibrium, those SNPs that had been associated with inflammatory traits $\left(\mathrm{p}<5 \times 10^{-8}\right)$, including those relating to cytokines, acute phase proteins, and immune cells, in previously published GWAS were subselected using the Phenoscanner $\mathrm{R}$ package ${ }^{39}$. These inflammatory-related IVs were used to further assess the role of inflammation in the association between smoking and depression.

CRP-cis SNPS:

When assessing the role of CRP, four variants - rs1205, rs3093077, rs1130864 and rs1800947 were selected as cis-variants, which are those located in the CRP gene region ${ }^{20}$. Limiting the analysis to cis-variants allows for more reliable conclusions due to the biological relevance of the variants used ${ }^{34}$. Clumping was performed using the $l d$ _clump() function from the ieugwasr $\mathrm{R}$ package (clumping window $\left.\mathrm{kb}=10000, r^{2}=0.001, \mathrm{p}=0.99\right)^{37}$ and the variant $\mathrm{rs} 3093077$ was selected as the lead CRP variant. Wald ratio MR analyses were performed to assess the effect of this variant on depression and smoking.

\section{Univariable reciprocal Mendelian randomization analysis}

The Inverse-variance weighted method was selected as the main method to calculate the combined effect of the selected instrumental variables in all univariable MR analyses. The IVW method is similar to a weighted regression of the effect of each specific IV on the outcome on the effect of the same IV on the exposure, restricting the intercept to zero ${ }^{40,41}$. All of the univariable MR analyses are represented in Figure 1 and in Table 3. The direction of the relationships was confirmed using Steiger filtering using the steiger_filtering() function from the TwoSampleMR $\mathrm{R}$ package $^{42-44}$. 
medRxiv preprint doi: https://doi.org/10.1101/2021.02.04.21251136; this version posted February 8, 2021. The copyright holder for this preprint (which was not certified by peer review) is the author/funder, who has granted medRxiv a license to display the preprint in perpetuity.

It is made available under a CC-BY 4.0 International license .

Fig. 1 Graphic representation of the different univariable MR analyses performed. The numbers for the different analyses correspond to those listed in Table 3.

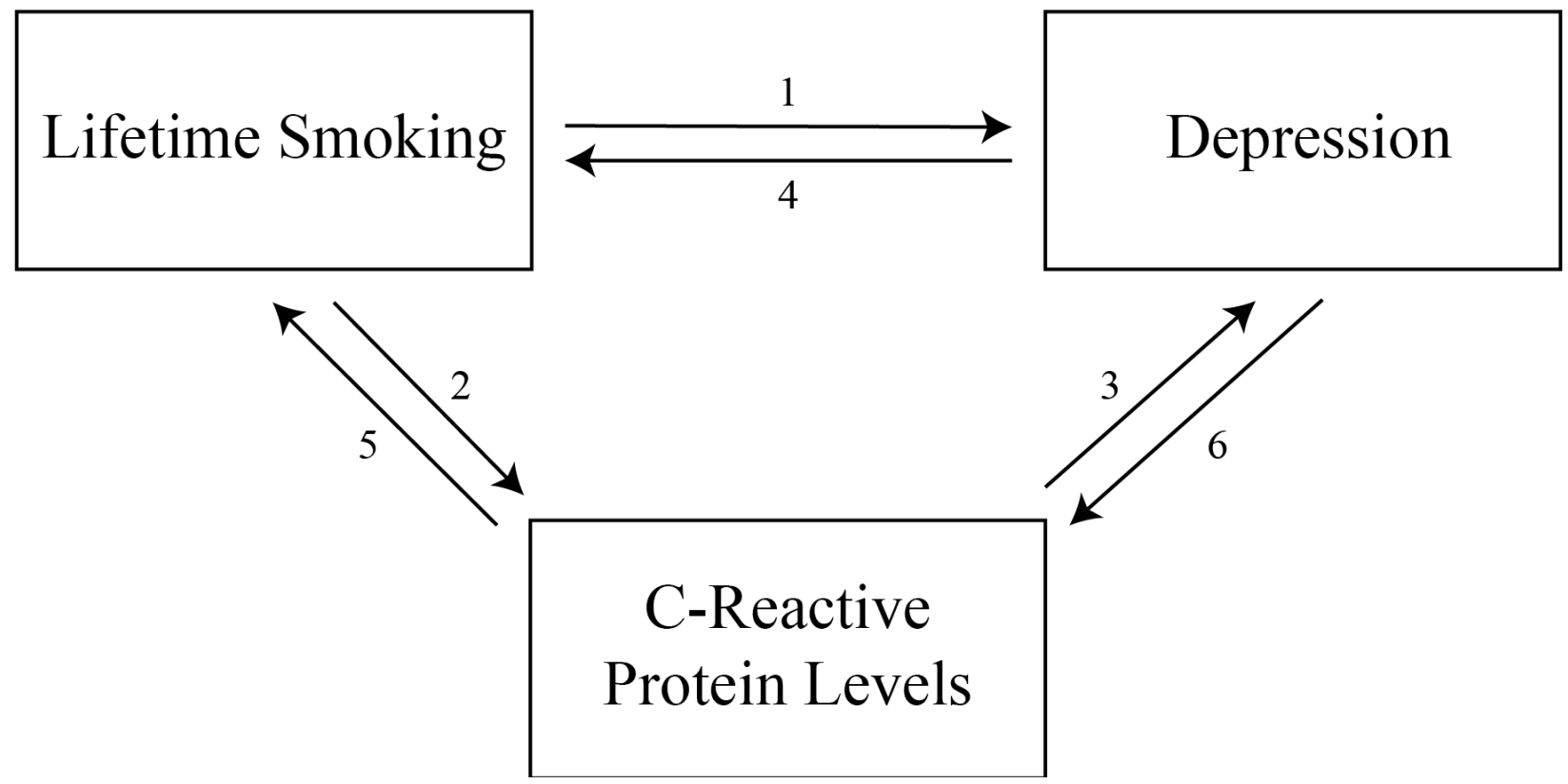

Table 3. Univariable MR analyses performed in this report. Analyses 1, 2, and 3 are the main analyses in this report and the rest are considered supplemental. Analyses 4, 5, and 6 are the reciprocal $M R$ analysis for 1, 2, and 3, respectively. Analyses 1b, 1c, and 3b include biological factors in the IV selection.

\begin{tabular}{|l|l|l|}
\hline ID & Exposure & Outcome \\
\hline 1 & Lifetime Smoking & Depression \\
\hline $1 \mathrm{~b}$ & $\begin{array}{l}\text { Lifetime Smoking - } \\
\text { Inflammation }\end{array}$ & Depression \\
\hline $1 \mathrm{c}$ & $\begin{array}{l}\text { Lifetime Smoking - non- } \\
\text { Inflammation }\end{array}$ & Depression \\
\hline 2 & Lifetime Smoking & CRP \\
\hline 3 & CRP & Depression \\
\hline 4 & Depression & Lifetime Smoking \\
\hline 5 & CRP & Smoking \\
\hline 6 & Depression & CRP \\
\hline
\end{tabular}

Heterogeneity and sensitivity analysis:

For all of the analyses performed, F-statistic were calculated to assess the strength of the instruments ${ }^{45}$. The heterogeneity between the estimates of the different IVs used was assessed using Cochran's Q, leave-one-out analyses, and visualizing the data with funnel, scatter, and 
forest plots ${ }^{44,46,47}$. Steiger filtering was used to assess the directionality of the relationship in the associations that were significant ${ }^{42}$.

The robustness of the overall estimate obtained with the IVW method was assessed using a combination of robust MR methods from different classes and working under a wide range of assumptions $^{42}$. Four additional MR methods were used: weighted median, MR-PRESSO, MREgger, and contamination mixture (Table 4). Weighted median is a median based estimate that provides valid estimate even if up to $50 \%$ of the instruments are invalid. MR-PRESSO accounts for pleiotropy by detecting and removing outliers. MR-Egger accounts for pleiotropy by including an intercept term in the IVW model. Contamination mixture accounts for heterogeneity in causal mechanisms by identifying genetic instruments with similar causal estimates.

Table 4. Summary comparison of the robust Mendelian randomization methods used in this report. Adapted from Slob et al. (2020).

\begin{tabular}{|l|l|l|l|}
\hline Method & Class & $\begin{array}{l}\text { Consistency } \\
\text { Assumptions }\end{array}$ & Majority valid \\
\hline Weighted median & Consensus & $\begin{array}{l}\text { Robust to outliers, } \\
\text { sensitive to } \\
\text { additional/ } \\
\text { removal of genetic } \\
\text { variants, may be less } \\
\text { efficient. }\end{array}$ \\
\hline MR-PRESSO & Outlier-robust & Outlier-robust & $\begin{array}{l}\text { Removes outliers, } \\
\text { efficient with valid } \\
\text { IVs, very high false } \\
\text { positive rate with } \\
\text { several invalid IVs. }\end{array}$ \\
\hline $\begin{array}{l}\text { Contamination } \\
\text { mixture }\end{array}$ & Modelling & Plurality valid & $\begin{array}{l}\text { Robust to outliers, } \\
\text { sensitive to variance } \\
\text { parameter and } \\
\text { addition/removal of } \\
\text { genetic variants. }\end{array}$ \\
\hline MR-Egger & Modelling & $\begin{array}{l}\text { Sensitive to outliers, } \\
\text { sensitive to violations } \\
\text { of InSIDE } \\
\text { assumption, InSIDE } \\
\text { assumption often not } \\
\text { plausible, may be less } \\
\text { efficient. }\end{array}$ \\
\hline
\end{tabular}

Multivariable Mendelian randomization analysis 
(MVMR) methods allow for the estimation of the proportion of the effect of smoking directly acting on depression and the proportion potentially being mediated by CRP levels - representing systemic inflammation. IV coefficients for smoking were regressed on the SNP coefficients for depression, followed by a second regression of the coefficients of the CRP on the previously obtained coefficients ${ }^{45}$. The global estimate is then produced using the IVW method. 


\section{$\underline{\text { Results }}$}

\section{Instrumental variable selection}

The results from the IV selection process, including all of the significant SNPs in each GWAS and the final IVs selected for each variable are shown in Table 5.

Table 5 Instrumental variable selection using statistical methods.

\begin{tabular}{|l|l|l|l|l|}
\hline Variable & GWAS SNPs & Significant SNPs & $\begin{array}{l}\text { Independents } \\
\text { SNPs }\end{array}$ & F-statistic $^{1}$ \\
\hline $\begin{array}{l}\text { Lifetime } \\
\text { Smoking }\end{array}$ & $7,683,352$ & 10,415 & 126 & 13.26 \\
\hline CRP & $8,927,092$ & 60,177 & 526 & 118.78 \\
\hline Depression & $8,484,301$ & 4,625 & 50 & 193.74 \\
\hline
\end{tabular}

${ }^{1}$ F-statistic values were estimated using previously reported $\mathrm{R}^{2}$ and may not represent the true value for the analyses included here.

\section{IVW MR Analyses Testing Association of Smoking with Depression and CRP}

The IVW method supported significant associations of genetically-predicted lifetime smoking index with risk of depression (ORSmk-Dep $=2.01,95 \% \mathrm{CI}: 1.71-2.37, \mathrm{p}<0.001$ ), and with CRP levels CRP (ORSmk-CRP $=1.40,95 \%$ CI : 1.27-1.55, $\mathrm{p}<0.001)$. The IVW method also identified a significant association between depression and lifetime smoking index (ORDep-Smk $=1.09,95 \%$ CI : 1.06-1.13, p $<0.001)$, and between genetically-predicted CRP levels and lifetime smoking index (ORCRP-Smk $=1.03,95 \%$ CI : 1.02-1.05, $\mathrm{p}<0.001)$ (Table 6). However, MR associations between CRP and depression were not statistically significant.

\section{MR Analysis using inflammation-related genetic variants for smoking as IVs}

Further analysis using the smoking-related genetic variants that are also associated with inflammation as IVs, the associations between smoking and depression remained significant for both inflammation-related and unrelated genetic variant sets (Table 6).

\section{MR Analysis using cis variants for CRP as IVs}

No association was observed between the lead CRP-cis variant, rs3093077, and depression or smoking (Table 6). 
medRxiv preprint doi: https://doi.org/10.1101/2021.02.04.21251136; this version posted February 8, 2021. The copyright holder for this preprint (which was not certified by peer review) is the author/funder, who has granted medRxiv a license to display the preprint in perpetuity.

It is made available under a CC-BY 4.0 International license .

Table 6 Inverse Variance Weighted Estimates for the univariable MR analyses.

\begin{tabular}{|l|l|l|l|l|l|l|l|}
\hline Exposure & Outcome & SNPs & OR & CI_low & CI_high & StdError & p-val \\
\hline Smoking & $\begin{array}{l}\text { Depressio } \\
\mathrm{n}\end{array}$ & 126 & 2.01 & 1.71 & 2.37 & 0.08 & $<0.001$ \\
\hline Smoking_Infl & $\begin{array}{l}\text { Depressio } \\
\mathrm{n}\end{array}$ & 15 & 1.89 & 1.23 & 2.91 & 0.22 & 0.004 \\
\hline $\begin{array}{l}\text { Smoking_nonIn } \\
\text { fl }\end{array}$ & $\begin{array}{l}\text { Depressio } \\
\mathrm{n}\end{array}$ & 114 & 2.07 & 1.73 & 2.47 & 0.09 & $<0.001$ \\
\hline Smoking & $\mathrm{CRP}$ & 126 & 1.4 & 1.27 & 1.55 & 0.05 & $<0.001$ \\
\hline CRP & $\begin{array}{l}\text { Depressio } \\
\mathrm{n}\end{array}$ & 512 & 1.01 & 0.99 & 1.04 & 0.01 & 0.225 \\
\hline CRP_cis* & $\begin{array}{l}\text { Depressio } \\
\mathrm{n}\end{array}$ & 1 & 1.00 & 0.92 & 1.09 & 0.04 & 1.000 \\
\hline Depression & CRP & 50 & 1.03 & 0.99 & 1.07 & 0.02 & 0.11 \\
\hline CRP & Smoking & 521 & 1.03 & 1.02 & 1.05 & 0.01 & $<0.001$ \\
\hline CRP_cis* & Smoking & 1 & 0.98 & 0.95 & 1.01 & 0.01 & 0.171 \\
\hline Depression & Smoking & 50 & 1.09 & 1.06 & 1.13 & 0.02 & $<0.001$ \\
\hline Abbreviatins: Smoking_Inf & & & & & & & \\
\hline
\end{tabular}

Abbreviations: Smoking_Infl indicates smoking IVs previously associated with inflammation. Smoking_nonInfl indicates smoking IVs not previously associated with inflammation. CI_low lower limit of 95\% confidence interval. CI_high upper limit of 95\% confidence interval.

*Wald ratios were used to obtain the estimate for the CRP_cis variant.

\section{Visual assessment of MR estimates}

Visual assessment of the individual IV estimates using scatter, funnel, and forest plots indicated the presence of moderate heterogeneity among these effect estimates (Figure $2 \&$ Supplemental Figures 1-3). However, the symmetrical distribution of individual estimates around the overall estimates suggests that the pleiotropy present is likely balanced. 
medRxiv preprint doi: https://doi.org/10.1101/2021.02.04.21251136; this version posted February 8, 2021. The copyright holder for this preprint (which was not certified by peer review) is the author/funder, who has granted medRxiv a license to display the preprint in perpetuity.

It is made available under a CC-BY 4.0 International license .

Figure 2. Scatter plots (left) and funnel plots (right) showing the individual IV estimates (dots) and the calculated global estimates (lines) in the main analyses.
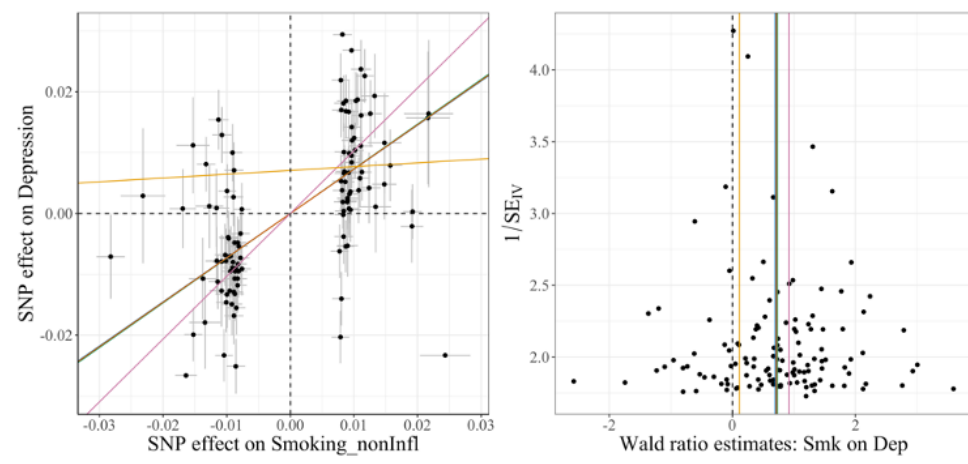

MR Test

IVW
Weighted Median

MR-PRESSO

Contamination Mixture
MR-Egger
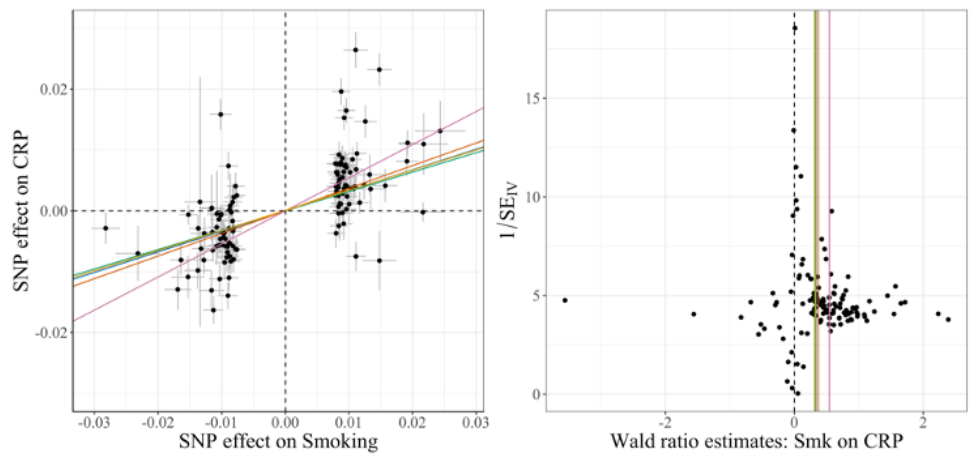

MR Test

IVW
Weighted Median

MR-PRESSO

Contamination Mixture
MR-Egger

Wald ratio estimates: Smk on CRP
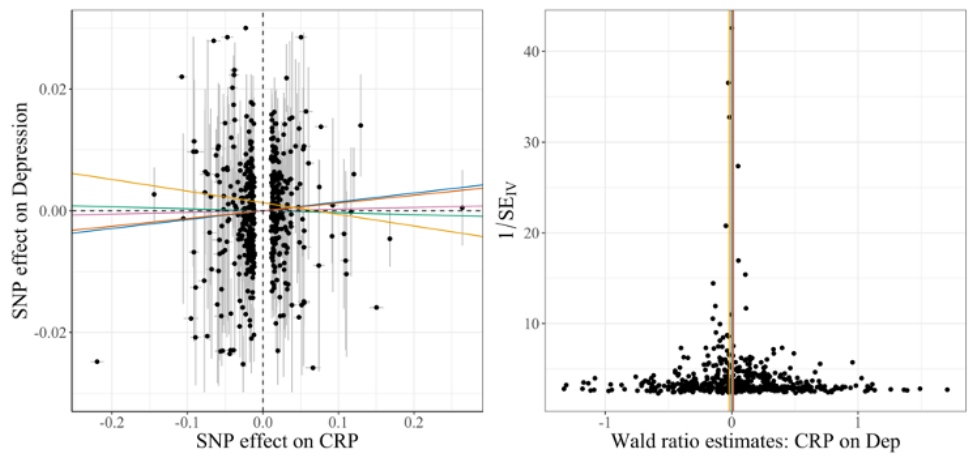

MR Test

IVW
Weighted Media

MR-PRESSO

Contamination Mixture

Wald ratio estimates: CRP on Dep

\section{Results for sensitivity analyses}

Influential IVS

Among the significant associations, every global estimate obtained through leave-one-out analyses remained significant (Supplemental Figure 2). Similarly, in non-significant associations, all of the global estimates obtained through leave-one-out analyses remained non-significant. This suggests that none of the IVs used throughout the analyses acted as an influential point.

\section{Robust analyses}

In the smoking-CRP, smoking-depression, and depression-smoking association, all of the global estimates calculated using robust MR methods, excluding the MR-Egger estimate, showed a 
similarly significant increase in the odds of the outcome and higher levels of the exposure. I2GX values calculated indicate that the MR-Egger is likely to provide biased estimates; furthermore, the QR values obtained do not support a better fit of the MR-Egger data to the model when compared to the IVW model (table 9).

Table 7. Robust MR Estimates for the univariable MR analyses.

\begin{tabular}{|c|c|c|c|c|c|c|c|c|}
\hline Exposure & Outcome & Method & $\begin{array}{l}\mathrm{N}_{\mathrm{SNP}} \\
\mathrm{s}\end{array}$ & OR & $\begin{array}{l}\text { StdErro } \\
\mathrm{r}\end{array}$ & \begin{tabular}{|l}
$95 \%$ \\
CI_Lo \\
w
\end{tabular} & $\begin{array}{l}95 \% \\
\text { CI } \\
\text { Uppe } \\
\mathrm{r}\end{array}$ & p-val \\
\hline Smoking & $\begin{array}{l}\text { Depressio } \\
\mathrm{n}\end{array}$ & IVW & 126 & $\begin{array}{l}2.0 \\
1\end{array}$ & 0.08 & 1.70 & 2.36 & $\begin{array}{l}<0.00 \\
1\end{array}$ \\
\hline Smoking & $\begin{array}{l}\text { Depressio } \\
\mathrm{n}\end{array}$ & $\begin{array}{l}\text { Weighted } \\
\text { Median }\end{array}$ & 126 & $\begin{array}{l}2.0 \\
7\end{array}$ & 0.08 & 1.79 & 2.41 & $\begin{array}{l}<0.00 \\
1\end{array}$ \\
\hline Smoking & $\begin{array}{l}\text { Depressio } \\
\mathrm{n}\end{array}$ & $\begin{array}{l}\text { MR- } \\
\text { PRESSO }\end{array}$ & 116 & $\begin{array}{l}2.0 \\
5\end{array}$ & 0.07 & 1.79 & 2.34 & $\begin{array}{l}<0.00 \\
1\end{array}$ \\
\hline Smoking & $\begin{array}{l}\text { Depressio } \\
\mathrm{n}\end{array}$ & $\begin{array}{l}\text { Contaminatio } \\
\mathrm{n}\end{array}$ & 79 & $\begin{array}{l}2.5 \\
1\end{array}$ & NA & 2.14 & 3.13 & $\begin{array}{l}<0.00 \\
1\end{array}$ \\
\hline Smoking & $\begin{array}{l}\text { Depressio } \\
\mathrm{n}\end{array}$ & MR-EGGER & 126 & $\begin{array}{l}1.1 \\
2\end{array}$ & 0.33 & 0.59 & 2.14 & 0.727 \\
\hline Smoking & CRP & IVW & 126 & $\begin{array}{l}1.4 \\
0\end{array}$ & 0.05 & 1.27 & 1.55 & $\begin{array}{l}<0.00 \\
1\end{array}$ \\
\hline Smoking & CRP & $\begin{array}{l}\text { Weighted } \\
\text { Median }\end{array}$ & 126 & $\begin{array}{l}1.3 \\
8\end{array}$ & 0.04 & 1.28 & 1.48 & $\begin{array}{l}<0.00 \\
1\end{array}$ \\
\hline Smoking & CRP & $\begin{array}{l}\text { MR- } \\
\text { PRESSO }\end{array}$ & 111 & $\begin{array}{l}1.4 \\
5\end{array}$ & 0.03 & 1.36 & 1.54 & $\begin{array}{l}<0.00 \\
1\end{array}$ \\
\hline Smoking & CRP & $\begin{array}{l}\text { Contaminatio } \\
\mathrm{n}\end{array}$ & 87 & $\begin{array}{l}1.7 \\
2\end{array}$ & NA & 1.63 & 1.80 & $\begin{array}{l}<0.00 \\
1\end{array}$ \\
\hline Smoking & CRP & MR-EGGER & 126 & $\begin{array}{l}1.3 \\
9 \\
\end{array}$ & 0.19 & 0.96 & 2.01 & 0.08 \\
\hline CRP & $\begin{array}{l}\text { Depressio } \\
\mathrm{n}\end{array}$ & IVW & 512 & $\begin{array}{l}1.0 \\
1\end{array}$ & 0.01 & 0.99 & 1.04 & 0.225 \\
\hline CRP & $\begin{array}{l}\text { Depressio } \\
\mathrm{n}\end{array}$ & $\begin{array}{l}\text { Weighted } \\
\text { Median }\end{array}$ & 512 & $\begin{array}{l}1.0 \\
0\end{array}$ & 0.02 & 0.97 & 1.03 & 0.838 \\
\hline CRP & $\begin{array}{l}\text { Depressio } \\
\mathrm{n}\end{array}$ & $\begin{array}{l}\text { MR- } \\
\text { PRESSO }\end{array}$ & 504 & $\begin{array}{l}1.0 \\
1\end{array}$ & 0.01 & 0.99 & 1.03 & 0.255 \\
\hline CRP & $\begin{array}{l}\text { Depressio } \\
\mathrm{n}\end{array}$ & $\begin{array}{l}\text { Contaminatio } \\
\mathrm{n}\end{array}$ & 380 & $\begin{array}{l}1.0 \\
0\end{array}$ & NA & 0.98 & 1.01 & 1 \\
\hline CRP & $\begin{array}{l}\text { Depressio } \\
\mathrm{n}\end{array}$ & MR-EGGER & 512 & $\begin{array}{l}0.9 \\
8\end{array}$ & 0.02 & 0.95 & 1.01 & 0.272 \\
\hline Depression & Smoking & IVW & 50 & $\begin{array}{l}1.0 \\
9 \\
\end{array}$ & 0.02 & 1.06 & 1.13 & $\begin{array}{l}<0.00 \\
1\end{array}$ \\
\hline
\end{tabular}




\begin{tabular}{|c|c|c|c|c|c|c|c|c|}
\hline Depression & Smoking & $\begin{array}{l}\text { Weighted } \\
\text { Median }\end{array}$ & 50 & $\begin{array}{l}1.1 \\
2 \\
\end{array}$ & 0.01 & 1.08 & 1.15 & $\begin{array}{l}<0.00 \\
1\end{array}$ \\
\hline Depression & Smoking & $\begin{array}{l}\text { MR- } \\
\text { PRESSO }\end{array}$ & 43 & $\begin{array}{l}1.1 \\
0\end{array}$ & 0.01 & 1.08 & 1.13 & $\begin{array}{l}<0.00 \\
1\end{array}$ \\
\hline Depression & Smoking & $\begin{array}{l}\text { Contaminatio } \\
\mathrm{n}\end{array}$ & 31 & $\begin{array}{l}1.1 \\
5 \\
\end{array}$ & NA & 1.13 & 1.19 & $\begin{array}{l}<0.00 \\
1\end{array}$ \\
\hline Depression & Smoking & MR-EGGER & 50 & $\begin{array}{l}1.0 \\
6\end{array}$ & 0.09 & 0.89 & 1.27 & 0.529 \\
\hline CRP & Smoking & IVW & 521 & $\begin{array}{l}1.0 \\
3 \\
\end{array}$ & 0.01 & 1.02 & 1.04 & $\begin{array}{l}<0.00 \\
1\end{array}$ \\
\hline CRP & Smoking & $\begin{array}{l}\text { Weighted } \\
\text { Median }\end{array}$ & 521 & $\begin{array}{l}1.0 \\
1\end{array}$ & 0.01 & 1.00 & 1.02 & 0.05 \\
\hline CRP & Smoking & $\begin{array}{l}\text { MR- } \\
\text { PRESSO }\end{array}$ & 493 & $\begin{array}{l}1.0 \\
3\end{array}$ & 0.00 & 1.02 & 1.04 & $\begin{array}{l}<0.00 \\
1\end{array}$ \\
\hline CRP & Smoking & $\begin{array}{l}\text { Contaminatio } \\
\mathrm{n}\end{array}$ & 349 & $\begin{array}{l}1.0 \\
1\end{array}$ & NA & 1.01 & 1.02 & 0.001 \\
\hline CRP & Smoking & MR-EGGER & 521 & $\begin{array}{l}1.0 \\
0\end{array}$ & 0.01 & 0.98 & 1.01 & 0.533 \\
\hline Depression & CRP & IVW & 50 & $\begin{array}{l}1.0 \\
3 \\
\end{array}$ & 0.02 & 0.99 & 1.06 & 0.11 \\
\hline Depression & CRP & $\begin{array}{l}\text { Weighted } \\
\text { Median }\end{array}$ & 50 & $\begin{array}{l}1.0 \\
0 \\
\end{array}$ & 0.01 & 0.98 & 1.03 & 0.856 \\
\hline Depression & CRP & $\begin{array}{l}\text { MR- } \\
\text { PRESSO }\end{array}$ & 44 & $\begin{array}{l}1.0 \\
4 \\
\end{array}$ & 0.01 & 1.01 & 1.06 & 0.009 \\
\hline Depression & CRP & $\begin{array}{l}\text { Contaminatio } \\
\mathrm{n}\end{array}$ & 26 & $\begin{array}{l}1.1 \\
7 \\
\end{array}$ & NA & 1.14 & 1.20 & 0.001 \\
\hline Depression & CRP & MR-EGGER & 50 & $\begin{array}{l}1.0 \\
5 \\
\end{array}$ & 0.10 & 0.85 & 1.28 & 0.642 \\
\hline Smoking_Infl & $\begin{array}{l}\text { Depressio } \\
\mathrm{n}\end{array}$ & IVW & 15 & $\begin{array}{l}1.8 \\
9\end{array}$ & 0.22 & 1.23 & 2.92 & 0.004 \\
\hline Smoking_Infl & $\begin{array}{l}\text { Depressio } \\
\mathrm{n}\end{array}$ & $\begin{array}{l}\text { Weighted } \\
\text { Median }\end{array}$ & 15 & $\begin{array}{l}1.9 \\
1\end{array}$ & 0.19 & 1.32 & 2.77 & 0.001 \\
\hline Smoking_Infl & $\begin{array}{l}\text { Depressio } \\
\mathrm{n}\end{array}$ & $\begin{array}{l}\text { MR- } \\
\text { PRESSO }\end{array}$ & 13 & $\begin{array}{l}1.8 \\
5\end{array}$ & 0.18 & 1.31 & 2.61 & 0.004 \\
\hline Smoking_Infl & $\begin{array}{l}\text { Depressio } \\
\mathrm{n}\end{array}$ & $\begin{array}{l}\text { Contaminatio } \\
\mathrm{n}\end{array}$ & 9 & $\begin{array}{l}2.0 \\
4\end{array}$ & NA & 1.34 & 3.10 & 0.012 \\
\hline Smoking_Infl & $\begin{array}{l}\text { Depressio } \\
\mathrm{n}\end{array}$ & MR-EGGER & 15 & $\begin{array}{l}2.1 \\
1 \\
\end{array}$ & 1.22 & 0.19 & 22.87 & 0.54 \\
\hline $\begin{array}{l}\text { Smoking_nonIn } \\
\text { fl }\end{array}$ & $\begin{array}{l}\text { Depressio } \\
\mathrm{n}\end{array}$ & IVW & 114 & $\begin{array}{l}2.0 \\
7 \\
\end{array}$ & 0.09 & 1.73 & 2.46 & $\begin{array}{l}<0.00 \\
1\end{array}$ \\
\hline $\begin{array}{l}\text { Smoking_nonIn } \\
\text { fl }\end{array}$ & $\begin{array}{l}\text { Depressio } \\
\mathrm{n}\end{array}$ & $\begin{array}{l}\text { Weighted } \\
\text { Median }\end{array}$ & 114 & $\begin{array}{l}2.0 \\
8 \\
\end{array}$ & 0.08 & 1.79 & 2.44 & $\begin{array}{l}<0.00 \\
1\end{array}$ \\
\hline
\end{tabular}




\begin{tabular}{|c|c|c|c|c|c|c|c|c|}
\hline $\begin{array}{l}\text { Smoking_nonIn } \\
\text { fl }\end{array}$ & $\begin{array}{l}\text { Depressio } \\
\mathrm{n}\end{array}$ & $\begin{array}{l}\text { MR- } \\
\text { PRESSO }\end{array}$ & 103 & $\begin{array}{l}2.0 \\
7\end{array}$ & 0.07 & 1.80 & 2.39 & $\begin{array}{l}<0.00 \\
1\end{array}$ \\
\hline $\begin{array}{l}\text { Smoking_nonIn } \\
\text { fl }\end{array}$ & $\begin{array}{l}\text { Depressio } \\
\mathrm{n}\end{array}$ & $\begin{array}{l}\text { Contaminatio } \\
\mathrm{n}\end{array}$ & 72 & $\begin{array}{l}2.8 \\
0\end{array}$ & NA & 2.14 & 3.56 & $\begin{array}{l}<0.00 \\
1\end{array}$ \\
\hline $\begin{array}{l}\text { Smoking_nonIn } \\
\text { fl }\end{array}$ & $\begin{array}{l}\text { Depressio } \\
\mathrm{n}\end{array}$ & MR-EGGER & 114 & $\begin{array}{l}1.0 \\
6 \\
\end{array}$ & 0.34 & 0.54 & 2.10 & 0.857 \\
\hline
\end{tabular}

Abbreviations: Smoking_Infl indicates smoking IVs previously associated with inflammation. Smoking_nonInfl indicates smoking IVs not previously associated with inflammation. CI_low lower limit of 95\% confidence interval. CI_high upper limit of 95\% confidence interval.

\section{Multivariable MR}

Using the IVW method, the MVMR analysis, using lifetime smoking as the exposure, estimated a significant OR of 2.08 for depression (95\% CI : 1.71-2.54; $<<0.001)$, and a non-significant OR of 0.92 for CRP levels (95\% CI : 0.71-1.19; $\mathrm{p}=0.542)$ (Table 8). The similarity between this estimate for smoking-depression and the unadjusted one, as well as the lack of significance for the CRP term in the model, does not provide evidence to support a role for CRP as a mediator the relationship between smoking and depression.

Table 8. MVMR Estimates.

\begin{tabular}{|c|c|c|c|c|c|c|c|c|}
\hline & Exposure & $\begin{array}{l}\text { Outco } \\
\text { me }\end{array}$ & nsnp & OR & $\begin{array}{l}\text { CILowe } \\
\mathrm{r}\end{array}$ & CIUpper & StdError & pval \\
\hline \multirow[t]{2}{*}{$\begin{array}{l}\text { Smk- } \\
\text { CRP-Dep }\end{array}$} & CRP & Dep & 10 & $\begin{array}{l}0.92172 \\
339\end{array}$ & $\begin{array}{l}0.70928 \\
808\end{array}$ & $\begin{array}{l}1.19778 \\
412\end{array}$ & $\begin{array}{l}0.13366 \\
5\end{array}$ & $\begin{array}{l}0.54198 \\
836\end{array}$ \\
\hline & Smk & Dep & 126 & $\begin{array}{l}2.08138 \\
803\end{array}$ & $\begin{array}{l}1.70563 \\
573\end{array}$ & $\begin{array}{l}2.53991 \\
872\end{array}$ & $\begin{array}{l}0.10158 \\
015\end{array}$ & $\begin{array}{l}5.34 \mathrm{E}- \\
13\end{array}$ \\
\hline \multirow[t]{2}{*}{$\begin{array}{l}\text { SmkInfl- } \\
\text { CRP-Dep }\end{array}$} & CRP & Dep & 4 & $\begin{array}{l}0.76394 \\
927\end{array}$ & $\begin{array}{l}0.46579 \\
656\end{array}$ & $\begin{array}{l}1.25294 \\
718\end{array}$ & $\begin{array}{l}0.25242 \\
47\end{array}$ & $\begin{array}{l}0.28612 \\
082\end{array}$ \\
\hline & Smk_Infl & Dep & 15 & $\begin{array}{l}2.12415 \\
896\end{array}$ & $\begin{array}{l}1.31593 \\
607\end{array}$ & $\begin{array}{l}3.42877 \\
695\end{array}$ & $\begin{array}{l}0.24429 \\
984\end{array}$ & $\begin{array}{l}0.00204 \\
363\end{array}$ \\
\hline \multirow[t]{2}{*}{$\begin{array}{l}\text { SmknonI } \\
\text { nfl-CRP- } \\
\text { Dep } \\
\end{array}$} & CRP & Dep & 7 & $\begin{array}{l}0.91312 \\
327\end{array}$ & $\begin{array}{l}0.68136 \\
585\end{array}$ & $\begin{array}{l}1.22370 \\
986\end{array}$ & $\begin{array}{l}0.14937 \\
321\end{array}$ & $\begin{array}{l}0.54289 \\
68\end{array}$ \\
\hline & $\begin{array}{l}\text { Smk_non } \\
\text { Infl }\end{array}$ & Dep & 114 & $\begin{array}{l}2.14868 \\
612\end{array}$ & $\begin{array}{l}1.73014 \\
326\end{array}$ & $\begin{array}{l}2.66847 \\
962\end{array}$ & $\begin{array}{l}0.11053 \\
69\end{array}$ & $\begin{array}{l}4.53 \mathrm{E}- \\
12\end{array}$ \\
\hline
\end{tabular}

Abbreviations: Smoking_Infl indicates smoking IVs previously associated with inflammation. Smoking_nonInfl indicates smoking IVs not previously associated with inflammation. NSNP number of SNPs.

\section{Inflammation-related smoking IVs}

Secondary MVMR analyses were conducted with the subset of inflammatory and noninflammatory smoking IVs being adjusted by CRP. These two analyses provided similar estimates to the ones obtained without adjusting by CRP levels (ORSmk-Infl $=2.12,95 \% \mathrm{CI}$ : 1.32-3.43, $\mathrm{p}=0.002$; ORSmk-nonInfl $=2.15,95 \% \mathrm{CI}: 1.73-2.67, \mathrm{p}<0.001)$ (Table 8). Again, these results do not support a role for CRP levels as a mediator for the relationship between smoking and depression. 
Assessment of bias and reliability of MR-Egger results

As MR-Egger tends to suffer from low statistical power and is particularly susceptible to bias from weak instruments ${ }^{47}$, we present assessment of reliability of the MR-Egger results (Table 9). $\mathrm{I}^{2}{ }_{\mathrm{GX}}$ for the data used in the different analyses (Table 9), suggested that the results from the MREgger analyses may be strongly biased and are not reliable; therefore, the IVW estimate is preferred $^{48,49}$.

Table 9. MR-Egger estimates for the main analyses and calculated I2gx and $Q$ values.

\begin{tabular}{|l|l|l|l|l|l|l|l|l|l|}
\hline $\begin{array}{l}\text { Expos } \\
\text { ure }\end{array}$ & $\begin{array}{l}\text { Outco } \\
\text { me }\end{array}$ & SNPs & OR & $\begin{array}{l}\text { StdErr } \\
\text { or }\end{array}$ & P-val & I2gx & Q' $^{\prime}$ & $\begin{array}{l}\text { Q's p- } \\
\text { val }\end{array}$ & Q_r \\
\hline $\begin{array}{l}\text { Smoki } \\
\text { ng }\end{array}$ & $\begin{array}{l}\text { Depres } \\
\text { sion }\end{array}$ & 126 & 1.12 & 0.33 & 0.727 & 0.4 & 488.92 & $<0.001$ & 0.974 \\
\hline $\begin{array}{l}\text { Smoki } \\
\text { ng }\end{array}$ & CRP & 126 & 1.21 & 0.33 & 0.08 & 0.98 & $\begin{array}{l}1139.6 \\
5\end{array}$ & $<0.001$ & 1 \\
\hline CRP & $\begin{array}{l}\text { Depres } \\
\text { sion }\end{array}$ & 512 & 0.98 & 0.02 & 0.272 & 0.98 & 992.31 & $<0.001$ & 0.986 \\
\hline
\end{tabular}


medRxiv preprint doi: https://doi.org/10.1101/2021.02.04.21251136; this version posted February 8, 2021. The copyright holder for this preprint (which was not certified by peer review) is the author/funder, who has granted medRxiv a license to display the preprint in perpetuity.

It is made available under a CC-BY 4.0 International license .

\section{Discussion:}

\section{Summary of findings:}

In this study, we present comprehensive Mendelian randomization analyses testing the direction and potential causality of association between smoking, CRP, and depression. Our results suggest potentially causal bi-directional associations of smoking with depression and CRP levels. However, there was no evidence for a potentially causal association between CRP levels and depression, or for a mediating role of inflammation for the association between smoking and depression. Results from multivariable Mendelian randomization analyses also suggest no evidence for the relationship between smoking and depression being mediated by CRP levels. The results obtained were consistent through the different sensitivity and robust analyses performed. Overall, the various robust methods performed relying on different assumptions, provide strong evidence to support the potential causal role of lifetime smoking on depression.

\section{Smoking and depression:}

For the relationship between smoking and depression, our results, using improved genetic instruments compared to prior studies, are consistent with previous evidence supporting a reciprocal association between these variables. Observational evidence has shown that depression can trigger smoking commencement and make its cessation more challenging ${ }^{50-54}$. Moreover, smoking has been shown to lead to depression, and smoking cessation has been associated with improved depressive outcomes and decreased depression symptomatology ${ }^{54-56}$. Wootton et al. (2019), using IVW methods and using data partially overlapping with that used in this study, obtained an OR of $1.99(95 \% \mathrm{CI}: 1.71-2.32 ; \mathrm{p}<0.001)$ for the causal role of lifetime smoking on depression, and an OR of 1.10 (95\% CI : 1.02-1.17; p $<0.001)$ for the reciprocal relationship between depression and smoking ${ }^{24}$. The main differences between this analysis and that in Wootton et al. (2019) is that the Howard et al (2019) depression GWAS that informed our study had a larger sample size and incorporated many cases with a broader depression definition, including broad depression (help-seeking for problems with nerves, anxiety, tension, or depression) and probable major depressive disorder. This broader definition allows for an increase in in statistical power resulting from the larger number of cases encompassed may result in a loss of specificity ${ }^{57}$. Nevertheless, the results obtained are very similar to those from Wootton et al. (2019) (we found an odds ratio of 2.01 for effect of smoking on depression compared to Wootton et al odds ratio of 1.99), suggesting that a despite the lack of a formal MDD diagnosis in all of the cases used, the broader depression definition used remains relevant for MR analysis ${ }^{27,29}$. The association between smoking and depression has important implications; it strengthens the case for primary prevention of smoking and stop-smoking initiatives, and raises the question, for future research, of whether smoking cessation initiatives are effective treatments for depression.

\section{Smoking and CRP:}

Evidence supporting a causal relationship between smoking and elevated CRP levels has been extensively documented in observational studies: with higher CRP levels in smokers ${ }^{13-15,58-71}$, and decreased CRP levels after smoking cessation ${ }^{14,15,58,63,65-68,70,71}$. This study provides new evidence to support a causal relationship between smoking behavior and CRP levels using Mendelian randomization techniques. Furthermore, this study supported a very small, but significant, effect (odds ratio of 1.03) of CRP levels on smoking lifetime index, which may be 
medRxiv preprint doi: https://doi.org/10.1101/2021.02.04.21251136; this version posted February 8, 2021. The copyright holder for this preprint (which was not certified by peer review) is the author/funder, who has granted medRxiv a license to display the preprint in perpetuity.

It is made available under a CC-BY 4.0 International license.

clinically negligible. Further study would be required to investigate which aspects of lifetime smoking CRP is causally associated with (as lifetime smoking is a composite measure reflecting both initiation and persistence of smoking).

\section{CRP and Depression:}

Our results did not support a potentially causal relationship between CRP and depression or vice versa, though several observational studies have demonstrated that patients with depression have significantly higher CRP levels when compared to those without depression ${ }^{26,31,72-75}$. Of previously published MR studies assessing this relationship ${ }^{20,25,26}$, our prior study (Khandaker et al. (2020)) found evidence for a potentially causal association between CRP levels and depression using data from the UK Biobank cohort. More recently, using the MR approach Kappelmann and colleagues have reported that inflammatory markers like CRP and IL-6 are associated with specific symptoms of depression, such as suicidality ${ }^{76}$. Using symptom-level data from the UK Biobank and Dutch NESDA cohorts we have reported observational and MR associations for CRP and IL-6 with somatic/neurovegetative symptoms of depression such as fatigue and sleeping difficulties ${ }^{77}$. Taken together, current evidence from epidemiological and genetic MR studies is consistent with inflammation being potentially causally related to certain symptoms of depression, namely somatic/neurovegetative symptoms, though MR evidence for a potentially causal role of CRP on the syndrome of depression as outcome is mixed.

\section{Inflammation as a mediator:}

The lack of difference in results of the analyses using inflammatory-related and noninflammatory related smoking IVs, suggest that there is no clear distinct CRP mediated inflammatory causal pathway mediating the causal relationship detected between smoking and depression. Furthermore, MVMR analyses showed that adjusting the effect of smoking on depression for CRP levels did not result in a significant estimate for the effect of CRP levels.

Overall, these results do not support a role for CRP-indexed inflammation in the development of depression. This is null effect contrasts with a prior finding, using the same CRP GWAS and a similar methodology, that serum CRP is causally associated with another multifactorial etiology phenotype - namely age-related macular degeneration - indicating that the genetic instruments we used are capable of revealing positive causal relationships between serum CRP and disease. Randomized control trials have shown that using of anti-inflammatory agents, such as celecoxib or infliximab, can successfully improve depression outcomes in patients with elevated CRP levels and patients with treatment-resistant depression ${ }^{78}$, strongly suggesting that inflammation has a crucial role in the pathogenesis of at least certain types of depression ${ }^{78,79}$. If inflammation is only causally relevant in certain subtypes of depression with their own distinct etiology and pathogenesis, our approach would not necessarily detect this. However, our results remain consistent with the possibility that inflammation may cause depression, considered as a unitary entity, via non-CRP mediated processes. Our research strongly suggests that future studies examining the effect of inflammatory cytokines on depression should broaden their scope beyond CRP. 
medRxiv preprint doi: https://doi.org/10.1101/2021.02.04.21251136; this version posted February 8, 2021. The copyright holder for this preprint (which was not certified by peer review) is the author/funder, who has granted medRxiv a license to display the preprint in perpetuity.

It is made available under a CC-BY 4.0 International license.

\section{Limitations:}

Lifetime smoking index and depression are both behavioral variables, with complex etiologies and hard to measure phenotypes. Furthermore, the statistical approach to IV selection use increases the risk of bias from horizontal pleiotropy ${ }^{80}$. However, the robust methods used, including MR-Egger, MR-PRESSO, and MR contamination mixture, allow MR analyses to be conducted in the presence of horizontal pleiotropy. The various sensitivity analyses we performed provide supporting evidence for the robustness of the findings.

It is important to note that all of the GWAS used for this study were obtained from the UK Biobank, resulting in a one-sample MR study. In one-sample MR studies, the exaggerated effect of the IVs on the exposure may lead to an overestimation of the causal effect between the exposure and the outcome assessed. This bias is called "winner's curse" and could result in a strong bias in the obtained results ${ }^{81,82}$. Nevertheless, previous studies performed in nonoverlapping samples have demonstrated the same direction of the effect between smoking and depression ${ }^{24}$. Furthermore, we used a smaller (47 independent IVs), non-overlapping CRP $\mathrm{GWAS}^{83}$, which showed a similar reciprocal associations between smoking and CRP, and CRP and depression (supplemental table $\mathrm{x}$ ).

\section{Conclusion}

The results from this study add on to the growing body of evidence supporting the bidirectionality of the causal relationship between smoking and depression. Furthermore, this study strengthens the evidence for a causal role of smoking on CRP levels. However, we do not find evidence for a potentially causal role for CRP on depression or for potentially mediating role for inflammation on the association between smoking and depression. Further research is needed to understand potential mechanisms for bidirectional association between smoking and depression. 
medRxiv preprint doi: https://doi.org/10.1101/2021.02.04.21251136; this version posted February 8, 2021. The copyright holder for this preprint (which was not certified by peer review) is the author/funder, who has granted medRxiv a license to display the preprint in perpetuity.

It is made available under a CC-BY 4.0 International license .

\section{Author Contribution/Credit Statement:}

Diego Galán: Conceptualization, Methodology, Formal Analysis, Writing- Original Draft. Ben Perry: Resources, Methodology, Writing- Review \& Editing. Varun Warrier: Conceptualization, Methodology, Writing- Review \& Editing. Golam Khandaker:

Methodology, Writing- Review \& Editing. Douglas Easton: Writing- Review \& Editing, Supervision. Graham Murray: Conceptualization, Methodology, Writing- Review \& Editing, Supervision.

\section{Funding:}

This work was supported by a Wellcome Trust award to GMK (201486/Z/16/Z), and a National Institute for Health Research award to BIP (DRF-2018-11-ST2-018). VW is supported by the Bowring Research Fellowship (St. Catharine's College, Cambridge) and a Wellcome Trust Collaborative Grant.

This work was supported by a Wellcome Trust award to GMK (201486 Z 16 Z) and a National Institute for Health Research award to BIP (DRF 201811 ST2 018). VW is supported by the Bowring Research Fellowship (St. Catharine's College Cambridge) and a Wellcome Trust Collaborative Grant

This work was supported by a Wellcome Trust award to GMK (201486 Z 16 Z) and a National Institute for Health Research award to BIP (DRF 201811 ST2 018). VW is supported by the Bowring Research Fellowship (St. Catharine's College Cambridge) and a Wellcome Trust Collaborative Grant

\section{Conflicts of Interest:}

All authors declare no conflicts of interest.

\section{$\underline{\text { References }}$}

1 Hashmi, A. M., Butt, Z. \& Umair, M. Is depression an inflammatory condition? A review of available evidence. J Pak Med Assoc 63, 899-906 (2013).

2 Miller, A. H. \& Raison, C. L. The role of inflammation in depression: from evolutionary imperative to modern treatment target. Nature Reviews Immunology 16, 22-34, doi:10.1038/nri.2015.5 (2016).

3 Banks, W. A. The blood-brain barrier in psychoneuroimmunology. Neurol Clin 24, 413419, doi:10.1016/j.ncl.2006.03.009 (2006).

4 Dunn, A. J., Wang, J. \& Ando, T. Effects of cytokines on cerebral neurotransmission. Comparison with the effects of stress. Adv Exp Med Biol 461, 117-127, doi:10.1007/9780-585-37970-8_8 (1999).

5 Konsman, J. P., Vigues, S., Mackerlova, L., Bristow, A. \& Blomqvist, A. Rat brain vascular distribution of interleukin-1 type-1 receptor immunoreactivity: relationship to patterns 
medRxiv preprint doi: https://doi.org/10.1101/2021.02.04.21251136; this version posted February 8, 2021. The copyright holder for this preprint (which was not certified by peer review) is the author/funder, who has granted medRxiv a license to display the preprint in perpetuity.

It is made available under a CC-BY 4.0 International license .

of inducible cyclooxygenase expression by peripheral inflammatory stimuli. J Comp Neurol 472, 113-129, doi:10.1002/cne.20052 (2004). cytokines in the pathophysiology of major depression. Biological psychiatry 65, 732-741, doi:10.1016/j.biopsych.2008.11.029 (2009).

7 Pace, T. W., Hu, F. \& Miller, A. H. Cytokine-effects on glucocorticoid receptor function: relevance to glucocorticoid resistance and the pathophysiology and treatment of major depression. Brain Behav Immun 21, 9-19, doi:10.1016/j.bbi.2006.08.009 (2007).

8 Vitkovic, L. et al. Cytokine signals propagate through the brain. Mol Psychiatry 5, 604615, doi:10.1038/sj.mp.4000813 (2000).

9 Gallo, J. J. et al. Depression, cardiovascular disease, diabetes, and two-year mortality among older, primary-care patients. Am J Geriatr Psychiatry 13, 748-755, doi:10.1176/appi.ajgp.13.9.748 (2005).

10 Jackson, J. L., DeZee, K. \& Berbano, E. Can treating depression improve disease outcomes? Ann Intern Med 140, 1054-1056, doi:10.7326/0003-4819-140-12200406150-00017 (2004).

11 Smith, P. H., Mazure, C. M. \& McKee, S. A. Smoking and mental illness in the U.S. population. Tob Control 23, e147-153, doi:10.1136/tobaccocontrol-2013-051466 (2014).

12 Gonçalves, R. B. et al. Impact of smoking on inflammation: overview of molecular mechanisms. Inflamm Res 60, 409-424, doi:10.1007/s00011-011-0308-7 (2011).

13 Arnson, Y., Shoenfeld, Y. \& Amital, H. Effects of tobacco smoke on immunity, inflammation and autoimmunity. J Autoimmun 34, J258-265, doi:10.1016/j.jaut.2009.12.003 (2010).

14 Bermudez, E. A., Rifai, N., Buring, J. E., Manson, J. E. \& Ridker, P. M. Relation between markers of systemic vascular inflammation and smoking in women. Am J Cardiol 89, 1117-1119, doi:10.1016/s0002-9149(02)02284-1 (2002).

15 Wannamethee, S. G. et al. Associations between cigarette smoking, pipe/cigar smoking, and smoking cessation, and haemostatic and inflammatory markers for cardiovascular disease. Eur Heart J 26, 1765-1773, doi:10.1093/eurheartj/ehi183 (2005).

16 Greenland, S. \& Morgenstern, H. Confounding in health research. Annu Rev Public Health 22, 189-212, doi:10.1146/annurev.publhealth.22.1.189 (2001).

17 Spieth, P. M. et al. Randomized controlled trials - a matter of design. Neuropsychiatr Dis Treat 12, 1341-1349, doi:10.2147/ndt.S101938 (2016).

18 Smith, G. D. et al. Clustered environments and randomized genes: a fundamental distinction between conventional and genetic epidemiology. PLoS Med 4, e352, doi:10.1371/journal.pmed.0040352 (2007).

19 Davies, N. M., Holmes, M. V. \& Davey Smith, G. Reading Mendelian randomisation studies: a guide, glossary, and checklist for clinicians. BMJ, k601, doi:10.1136/bmj.k601 (2018).

20 Khandaker, G. M. et al. Shared mechanisms between coronary heart disease and depression: findings from a large UK general population-based cohort. Molecular Psychiatry 25, 1477-1486, doi:10.1038/s41380-019-0395-3 (2020). 
medRxiv preprint doi: https://doi.org/10.1101/2021.02.04.21251136; this version posted February 8, 2021. The copyright holder for this preprint (which was not certified by peer review) is the author/funder, who has granted medRxiv a license to display the preprint in perpetuity. It is made available under a CC-BY 4.0 International license.

21 Lawlor, D. A., Harbord, R. M., Sterne, J. A. C., Timpson, N. \& Davey Smith, G. Mendelian randomization: Using genes as instruments for making causal inferences in epidemiology. Statistics in Medicine 27, 1133-1163, doi:10.1002/sim.3034 (2008).

22 Smith, G. D. \& Ebrahim, S. 'Mendelian randomization': can genetic epidemiology contribute to understanding environmental determinants of disease? Int J Epidemiol 32, 1-22, doi:10.1093/ije/dyg070 (2003).

23 Thanassoulis, G. \& O'Donnell, C. J. Mendelian randomization: nature's randomized trial in the post-genome era. Jama 301, 2386-2388, doi:10.1001/jama.2009.812 (2009).

24 Wootton, R. E. et al. Evidence for causal effects of lifetime smoking on risk for depression and schizophrenia: a Mendelian randomisation study. Psychol Med, 1-9, doi:10.1017/s0033291719002678 (2019).

25 Dardani, C. et al. Disentangling causal relationships between inflammatory markers and depression: a bidirectional Mendelian randomization analysis. bioRxiv, 712133, doi:10.1101/712133 (2019).

26 Wium-Andersen, M. K., Ørsted, D. D. \& Nordestgaard, B. G. Elevated C-Reactive Protein, Depression, Somatic Diseases, and All-Cause Mortality: A Mendelian Randomization Study. Biological Psychiatry 76, 249-257, doi:https://doi.org/10.1016/j.biopsych.2013.10.009 (2014).

27 Howard, D.M., Adams, M.J., Clarke, TK. et al. Genome-wide meta-analysis of depression identifies 102 independent variants and highlights the importance of the prefrontal brain regions. Nat Neurosci 22, 343-352 (2019).

28 Han, X. et al. Using Mendelian randomization to evaluate the causal relationship between serum C-reactive protein levels and age-related macular degeneration. Eur J Epidemiol 35, 139-146, doi:10.1007/s10654-019-00598-z (2020).

29 Wray, N. R. et al. Genome-wide association analyses identify 44 risk variants and refine the genetic architecture of major depression. Nat Genet 50, 668-681, doi:10.1038/s41588-018-0090-3 (2018).

30 Ripke, S. et al. A mega-analysis of genome-wide association studies for major depressive disorder. Mol Psychiatry 18, 497-511, doi:10.1038/mp.2012.21 (2013).

31 Gimeno, D. et al. Associations of C-reactive protein and interleukin- 6 with cognitive symptoms of depression: 12-year follow-up of the Whitehall II study. Psychological medicine 39, 413-423, doi:10.1017/S0033291708003723 (2009).

32 Tibuakuu, M. et al. The association between cigarette smoking and inflammation: The Genetic Epidemiology Network of Arteriopathy (GENOA) study. PLoS One 12, e0184914, doi:10.1371/journal.pone.0184914 (2017).

33 Fadista, J., Manning, A. K., Florez, J. C. \& Groop, L. The (in)famous GWAS P-value threshold revisited and updated for low-frequency variants. European Journal of Human Genetics 24, 1202-1205, doi:10.1038/ejhg.2015.269 (2016).

34 Burgess, S. et al. Guidelines for performing Mendelian randomization investigations [version 2; peer review: 2 approved]. Wellcome Open Research 4, doi:10.12688/wellcomeopenres.15555.2 (2020).

35 Burgess, S., Dudbridge, F. \& Thompson, S. G. Combining information on multiple instrumental variables in Mendelian randomization: comparison of allele score and 
medRxiv preprint doi: https://doi.org/10.1101/2021.02.04.21251136; this version posted February 8, 2021. The copyright holder for this preprint (which was not certified by peer review) is the author/funder, who has granted medRxiv a license to display the preprint in perpetuity.

It is made available under a CC-BY 4.0 International license.

summarized data methods. Statistics in medicine 35, 1880-1906, doi:10.1002/sim.6835 (2016).

36 Swerdlow, D. I. et al. Selecting instruments for Mendelian randomization in the wake of genome-wide association studies. International Journal of Epidemiology 45, 1600-1616, doi:10.1093/ije/dyw088 (2016).

37 Hemani, G. ieugwasr: R interface to the IEU GWAS database API. $R$ package (2020).

38 Myers, T. A., Chanock, S. J. \& Machiela, M. J. LDlinkR: An R Package for Rapidly Calculating Linkage Disequilibrium Statistics in Diverse Populations. Frontiers in Genetics 11, doi:10.3389/fgene.2020.00157 (2020).

39 Kamat, M. A. et al. PhenoScanner V2: an expanded tool for searching human genotypephenotype associations. Bioinformatics 35, 4851-4853, doi:10.1093/bioinformatics/btz469 (2019).

40 Burgess, S., Butterworth, A. \& Thompson, S. G. Mendelian Randomization Analysis With Multiple Genetic Variants Using Summarized Data. Genetic Epidemiology 37, 658-665, doi:10.1002/gepi.21758 (2013).

41 Gage, S. H. et al. Assessing causality in associations between cannabis use and schizophrenia risk: a two-sample Mendelian randomization study. Psychological Medicine 47, 971-980, doi:10.1017/S0033291716003172 (2017).

42 Hemani, G. et al. Automating Mendelian randomization through machine learning to construct a putative causal map of the human phenome. bioRxiv, 173682, doi:10.1101/173682 (2017).

43 Hemani, G., Tilling, K. \& Davey Smith, G. Orienting the causal relationship between imprecisely measured traits using GWAS summary data. PLOS Genetics 13, e1007081, doi:10.1371/journal.pgen.1007081 (2017).

44 Hemani, G. et al. The MR-Base platform supports systematic causal inference across the human phenome. elife 7, e34408, doi:10.7554/eLife.34408 (2018).

45 Burgess, S. \& Thompson, S. G. Multivariable Mendelian Randomization: The Use of Pleiotropic Genetic Variants to Estimate Causal Effects. American Journal of Epidemiology 181, 251-260, doi:10.1093/aje/kwu283 (2015).

46 Burgess, S., Bowden, J., Fall, T., Ingelsson, E. \& Thompson, S. G. Sensitivity Analyses for Robust Causal Inference from Mendelian Randomization Analyses with Multiple Genetic Variants. Epidemiology 28, 30-42, doi:10.1097/EDE.0000000000000559 (2017).

47 Zheng, J. et al. Recent Developments in Mendelian Randomization Studies. Current Epidemiology Reports 4, 330-345, doi:10.1007/s40471-017-0128-6 (2017).

48 Bowden, J. et al. Assessing the suitability of summary data for two-sample Mendelian randomization analyses using MR-Egger regression: the role of the 12 statistic. Int J Epidemiol 45, 1961-1974, doi:10.1093/ije/dyw220 (2016).

49 Burgess, S. \& Thompson, S. G. Interpreting findings from Mendelian randomization using the MR-Egger method. Eur J Epidemio/ 32, 377-389, doi:10.1007/s10654-017-0255-x (2017).

50 Akambase, J. A. et al. Depression Outcomes in Smokers and Nonsmokers: Comparison of Collaborative Care Management Versus Usual Care. J Prim Care Community Health 10, 2150132719861265, doi:10.1177/2150132719861265 (2019). 
medRxiv preprint doi: https://doi.org/10.1101/2021.02.04.21251136; this version posted February 8, 2021. The copyright holder for this preprint (which was not certified by peer review) is the author/funder, who has granted medRxiv a license to display the preprint in perpetuity.

It is made available under a CC-BY 4.0 International license.

51 Breslau, N., Kilbey, M. M. \& Andreski, P. Nicotine dependence and major depression. New evidence from a prospective investigation. Arch Gen Psychiatry 50, 31-35, doi:10.1001/archpsyc.1993.01820130033006 (1993).

52 Hitsman, B. et al. Past major depression and smoking cessation outcome: a systematic review and meta-analysis update. Addiction 108, 294-306, doi:10.1111/add.12009 (2013).

53 Kendler, K. S. et al. Smoking and major depression. A causal analysis. Arch Gen Psychiatry 50, 36-43, doi:10.1001/archpsyc.1993.01820130038007 (1993).

54 Stepankova, L. et al. Depression and Smoking Cessation: Evidence from a Smoking Cessation Clinic with 1-Year Follow-Up. Annals of Behavioral Medicine 51, 454-463, doi:10.1007/s12160-016-9869-6 (2017).

55 Rodríguez-Cano, R. et al. Smoking cessation and depressive symptoms at 1-, 3-, 6-, and 12-months follow-up. J Affect Disord 191, 94-99, doi:10.1016/j.jad.2015.11.042 (2016).

56 Taylor, G. et al. Change in mental health after smoking cessation: systematic review and meta-analysis. BMJ : British Medical Journal 348, g1151, doi:10.1136/bmj.g1151 (2014).

57 Cai, N. et al. Minimal phenotyping yields genome-wide association signals of low specificity for major depression. Nature Genetics 52, 437-447, doi:10.1038/s41588-0200594-5 (2020).

58 Bakhru, A. \& Erlinger, T. P. Smoking cessation and cardiovascular disease risk factors: results from the Third National Health and Nutrition Examination Survey. PLoS Med 2, e160, doi:10.1371/journal.pmed.0020160 (2005).

59 Bazzano, L. A., He, J., Muntner, P., Vupputuri, S. \& Whelton, P. K. Relationship between cigarette smoking and novel risk factors for cardiovascular disease in the United States. Ann Intern Med 138, 891-897, doi:10.7326/0003-4819-138-11-200306030-00010 (2003).

60 Collaboration, T. E. R. F. C-reactive protein concentration and risk of coronary heart disease, stroke, and mortality: an individual participant meta-analysis. The Lancet 375, 132-140, doi:https://doi.org/10.1016/S0140-6736(09)61717-7 (2010).

61 Conen, D. et al. Smoking, smoking cessation, [corrected] and risk for symptomatic peripheral artery disease in women: a cohort study. Ann Intern Med 154, 719-726, doi:10.7326/0003-4819-154-11-201106070-00003 (2011).

62 Eugen-Olsen, J., Ladelund, S. \& Sørensen, L. T. Plasma suPAR is lowered by smoking cessation: a randomized controlled study. Eur J Clin Invest 46, 305-311, doi:10.1111/eci.12593 (2016).

63 Hastie, C. E., Haw, S. \& Pell, J. P. Impact of smoking cessation and lifetime exposure on C-reactive protein. Nicotine Tob Res 10, 637-642, doi:10.1080/14622200801978722 (2008).

64 Kawada, T. Relationships between the smoking status and plasma fibrinogen, white blood cell count and serum C-reactive protein in Japanese workers. Diabetes \& Metabolic Syndrome: Clinical Research \& Reviews 9, 180-182, doi:https://doi.org/10.1016/j.dsx.2015.02.010 (2015).

65 Lowe, G. D., Yarnell, J. W., Rumley, A., Bainton, D. \& Sweetnam, P. M. C-reactive protein, fibrin D-dimer, and incident ischemic heart disease in the Speedwell study: are inflammation and fibrin turnover linked in pathogenesis? Arterioscler Thromb Vasc Biol 21, 603-610, doi:10.1161/01.atv.21.4.603 (2001). 
medRxiv preprint doi: https://doi.org/10.1101/2021.02.04.21251136; this version posted February 8, 2021. The copyright holder for this preprint (which was not certified by peer review) is the author/funder, who has granted medRxiv a license to display the preprint in perpetuity.

It is made available under a CC-BY 4.0 International license .

66 Ohsawa, M. et al. CRP levels are elevated in smokers but unrelated to the number of cigarettes and are decreased by long-term smoking cessation in male smokers. Prev Med 41, 651-656, doi:10.1016/j.ypmed.2005.02.002 (2005).

67 Shiels, M. S. et al. Cigarette Smoking and Variations in Systemic Immune and Inflammation Markers. JNCl: Journal of the National Cancer Institute 106, doi:10.1093/jnci/dju294 (2014).

68 Tonstad, S. \& Cowan, J. L. C-reactive protein as a predictor of disease in smokers and former smokers: a review. Int J Clin Pract 63, 1634-1641, doi:10.1111/j.17421241.2009.02179.x (2009).

69 Lao, X. Q. et al. Smoking, smoking cessation and inflammatory markers in older Chinese men: The Guangzhou Biobank Cohort Study. Atherosclerosis 203, 304-310, doi:10.1016/j.atherosclerosis.2008.06.028 (2009).

70 McEvoy John, W. et al. Relationship of Cigarette Smoking With Inflammation and Subclinical Vascular Disease. Arteriosclerosis, Thrombosis, and Vascular Biology 35, 1002-1010, doi:10.1161/ATVBAHA.114.304960 (2015).

71 Gallus, S. et al. Effect of Tobacco Smoking Cessation on C-Reactive Protein Levels in A Cohort of Low-Dose Computed Tomography Screening Participants. Scientific Reports 8, 12908, doi:10.1038/s41598-018-29867-9 (2018).

72 Dowlati, Y. et al. A meta-analysis of cytokines in major depression. Biol Psychiatry 67, 446-457, doi:10.1016/j.biopsych.2009.09.033 (2010).

73 Haapakoski, R., Mathieu, J., Ebmeier, K. P., Alenius, H. \& Kivimäki, M. Cumulative metaanalysis of interleukins 6 and $1 \beta$, tumour necrosis factor $\alpha$ and C-reactive protein in patients with major depressive disorder. Brain Behav Immun 49, 206-215, doi:10.1016/j.bbi.2015.06.001 (2015).

74 Howren, M. B., Lamkin, D. M. \& Suls, J. Associations of depression with C-reactive protein, IL-1, and IL-6: a meta-analysis. Psychosom Med 71, 171-186, doi:10.1097/PSY.0b013e3181907c1b (2009).

75 Valkanova, V., Ebmeier, K. P. \& Allan, C. L. CRP, IL-6 and depression: A systematic review and meta-analysis of longitudinal studies. Journal of Affective Disorders 150, 736-744, doi:https://doi.org/10.1016/j.jad.2013.06.004 (2013).

76 Kappelmann, N. et al. Dissecting the Association Between Inflammation, Metabolic Dysregulation, and Specific Depressive Symptoms. JAMA Psychiatry, doi:10.1001/jamapsychiatry.2020.3436 (2020).

77 Milaneschi Y, K. N., Lamers F, Moser S, Jones PB, Burgess S, Pennix BWJH, Khandaker GM. Association of Inflammation with Depression and Anxiety: Evidence for SymptomSpecificity and Potential Causality from UK Biobank and NESDA Cohorts. (Under Review) (2021).

78 Raison, C. L. et al. A randomized controlled trial of the tumor necrosis factor antagonist infliximab for treatment-resistant depression: the role of baseline inflammatory biomarkers. JAMA Psychiatry 70, 31-41, doi:10.1001/2013.jamapsychiatry.4 (2013).

79 Kohler, O., Krogh, J., Mors, O. \& Benros, M. E. Inflammation in Depression and the Potential for Anti-Inflammatory Treatment. Curr Neuropharmacol 14, 732-742, doi:10.2174/1570159x14666151208113700 (2016). 
80 Koellinger, P. D. \& De Vlaming, R. Mendelian randomization: the challenge of unobserved environmental confounds. International Journal of Epidemiology 48, 665671, doi:10.1093/ije/dyz138 (2019).

81 Haycock, P. C. et al. Best (but oft-forgotten) practices: the design, analysis, and interpretation of Mendelian randomization studies. Am J Clin Nutr 103, 965-978, doi:10.3945/ajcn.115.118216 (2016).

82 Taylor, A. E. et al. Mendelian randomization in health research: Using appropriate genetic variants and avoiding biased estimates. Economics \& Human Biology 13, 99-106, doi:https://doi.org/10.1016/i.ehb.2013.12.002 (2014).

83 Ligthart, S. et al. Genome Analyses of $>200,000$ Individuals Identify 58 Loci for Chronic Inflammation and Highlight Pathways that Link Inflammation and Complex Disorders. Am J Hum Genet 103, 691-706, doi:10.1016/j.ajhg.2018.09.009 (2018). 